\title{
The relationship between diabetes and clinical outcomes in COVID-19: a single-center retrospective analysis
}

\author{
Tamaryn Fox ${ }^{1}$ (D) Kathleen Ruddiman ${ }^{1} \cdot$ Kevin Bryan Lo ${ }^{1}$ Eric Peterson ${ }^{1} \cdot$ Robert DeJoy III ${ }^{1}$. \\ Grace Salacup ${ }^{1}$. Jerald Pelayo ${ }^{1}$. Ruchika Bhargav ${ }^{1}$. Fahad Gul ${ }^{1}$. Jeri Albano ${ }^{1}$ ' Zurab Azmaiparashvili ${ }^{1}$. \\ Catherine Anastasopoulou ${ }^{1,2,3} \cdot$ Gabriel Patarroyo-Aponte ${ }^{1,3,4}$
}

Received: 30 June 2020 / Accepted: 5 August 2020 / Published online: 17 August 2020

(c) Springer-Verlag Italia S.r.l., part of Springer Nature 2020

\begin{abstract}
Aims Coronavirus disease 19 (COVID-19) has become a pandemic. Diabetic patients tend to have poorer outcomes and more severe disease (Kumar et al. in Diabetes Metab Syndr 14(4):535-545, 2020. https://doi.org/10.1016/j.dsx.2020.04.044). However, the vast majority of studies are representative of Asian and Caucasian population and fewer represent an AfricanAmerican population.

Methods In this single-center, retrospective observational study, we included all adult patients ( $>18$ years old) admitted to Einstein Medical Center, Philadelphia, with a diagnosis of COVID-19. Patients were classified according to having a known diagnosis of diabetes mellitus. Demographic and clinical data, comorbidities, outcomes and laboratory findings were obtained.

Results Our sample included a total of 355 patients. $70 \%$ were African-American, and $47 \%$ had diabetes. Patients with diabetes had higher peak inflammatory markers like CRP 184 (111-258) versus 142 (65-229) $p=0.012$ and peak LDH 560 (384-758) versus 499 (324-655) $p=0.017$. The need for RRT/HD was significantly higher in patients with diabetes $(21 \%$ vs $11 \% p=0.013)$ as well as the need for vasopressors ( $28 \%$ vs $18 \% p=0.023)$. Only age was found to be an independent predictor of mortality. We found no significant differences in inpatient mortality $p=0.856$, need for RRT/HD $p=0.429$, need for intubation $p=1.000$ and need for vasopressors $p=0.471$ in African-Americans with diabetes when compared to non-African-Americans.

Conclusions Our study demonstrates that patients with COVID-19 and diabetes tend to have more severe disease and poorer clinical outcomes. African-American patients with diabetes did not differ in outcomes or disease severity when compared to non-African-American patients.
\end{abstract}

Keywords COVID-19 $\cdot$ Diabetes $\cdot$ Mortality $\cdot$ Novel coronavirus $\cdot$ Outcomes

\section{Introduction}

Managed by Massimo Porta.

Tamaryn Fox

FoxTamar@einstein.edu

1 Department of Medicine, Einstein Medical Center Philadelphia, 5501 Old York Road, Philadelphia, PA 19141, USA

2 Department of Endocrinology, Einstein Medical Center Philadelphia, Philadelphia, USA

3 Sidney Kimmel College of Thomas Jefferson University, Philadelphia, PA, USA

4 Pulmonary, Critical Care and Sleep Medicine, Einstein Medical Center Philadelphia, Philadelphia, USA
A cluster of pneumonia cases of unclear etiology originating in Wuhan city, Hubei Province, China, in late December 2019 now known as Coronavirus Disease 2019 (COVID-19) has become a pandemic [2]. It has affected over 1.9 million people in the USA alone [3] and 7.2 million people worldwide [4].

Reviewing literature on the effect of having diabetes in the context of other respiratory viral syndromes such as Middle Eastern Respiratory Syndrome (MERS) and Severe Acute Respiratory Syndrome (SARS) shows that patients with diabetes have been shown to have higher mortality 
rates $[5,6]$. This demonstrates the importance of reviewing the impact of diabetes in context of COVID-19.

COVID-19 outcomes tend to be poorer in patients with advanced age and multiple comorbidities [7, 8] Diabetes mellitus in general is widely known to cause significant morbidity and mortality as well as healthcare expenditure [9]. A meta-analysis including 33 studies inclusive of 16,000 patients investigated the relationship between COVID-19 and diabetes. It found that those with diabetes had higher risk of severe disease as well as higher rates of mortality [1]. Important to note that in this meta-analysis, the majority of patients were Asian or Caucasian, 30 of the 33 studies were from China, one in France and two in the USA.

Thus, it becomes imperative amidst the current COVID19 pandemic to investigate the interplay between diabetes and COVID-19 to potentially elucidate treatment strategies and further research opportunities in this specific population. In our study, we were able to highlight a high-risk, predominantly African-American population with multiple comorbidities. This gave us the unique opportunity to see if demographic data, comorbidities and other characteristics including disease severity and outcomes differed in those hospitalized with COVID-19 with and without a diagnosis of diabetes mellitus.

\section{Methods}

\section{Study design, participants and data collection}

This study was a single-center retrospective analysis of all patients 18 years of age or older who were admitted to Einstein Medical Center, Philadelphia, from March 1 to April 24, 2020, with a confirmed diagnosis of COVID-19 via reverse transcriptase-polymerase chain reaction assays (RT-PCR) performed on nasopharyngeal swab specimens. Laboratory values were collected including D-dimer (by Stago Compact Max), ferritin (by Architect I2000 SR Immunoassay), CRP (by Architect C8000 Clinical Chemistry), procalcitonin (by Architect I2000 SR Immunoassay), LDH (by Architect C 8000 Clinical Chemistry) and hemoglobin A1c (by Architect C 8000 Clinical Chemistry). Patients were classified according to having a known diagnosis of diabetes mellitus. We included patients with pharmacologic treatment of diabetes before admission consisting of a broad range of oral hypoglycemic agents, injectable agents and insulin. Pharmacological treatment of diabetes during hospitalization consisted mainly of insulin, while oral hypoglycemic agents were suspended. Demographic and clinical data, comorbidities, outcomes and laboratory findings were obtained. This study was approved by the Institutional Review Board.

\section{Statistical analysis}

Demographic variables were presented using descriptive statistics and frequencies. Categorical variables were analyzed with chi-square testing. Demographic and clinical variables were tabulated. Independent $t$-test was used for continuous variables. For skewed variables, Mann-Whitney $U$ test was used to compare differences. Outcomes such as inpatient death, need for renal replacement therapy or hemodialysis (RRT/HD) and need for vasopressors or intubation were considered. Multivariate logistic regression was used to evaluate the factors associated with mortality among patients with diabetes and COVID-19.95\% confidence intervals were used and are presented when appropriate. All analyses were performed using IBM's SPSS Statistics for Windows, Version 23.0.

\section{Results}

\section{Demographic and clinical characteristics of the patients}

A total of 389 patients were evaluated in our hospital and tested positive via RT-PCR for COVID-19. Nine patients were excluded who were still admitted at the time of analysis. Twenty-five patients were excluded due to incomplete clinical outcome data, leaving a final sample of 355 patients (see Fig. 1). In the final sample of 355 patients, the mean age $( \pm \mathrm{SD})$ was $66.21 \pm 14.21,49 \%$ were female and $70 \%$ were African-American. Chronic medical conditions of these patients included hypertension (77\%), diabetes mellitus (47\%), COPD (13\%) and asthma (8\%). The number of in hospital deaths was $80(23 \%)$. The mean HbA1c among patients with diabetes was $7.84 \pm 2.33$.

Traditional cardiovascular risk factors such as hypertension $(91 \%$ vs $64 \% p<0.0001)$ and chronic kidney disease $(25 \%$ vs $12 \% p=0.002)$ were higher in patients with diabetes compared to those without. Body mass index (BMI) was also significantly higher in patients with diabetes $(31.1 \pm 8.5$ vs $28.5 \pm 9.4 p=0.009)$ compared to those without. Cardiovascular disease such as heart failure $(24 \%$ vs $11 \% p=0.001)$ and coronary artery disease ( $27 \%$ vs $17 \% p=0.028)$ were more frequently present in patients with diabetes. During the hospital course, patients with diabetes had a significantly higher peak CRP 184 (111-258) versus $142(65-229) p=0.012$ and peak LDH $560(384-758)$ versus 499 (324-655) $p=0.017$. Meanwhile, other inflammatory markers such as ferritin and procalcitonin showed trends toward significance (see Table 1). There were significantly more patients with 


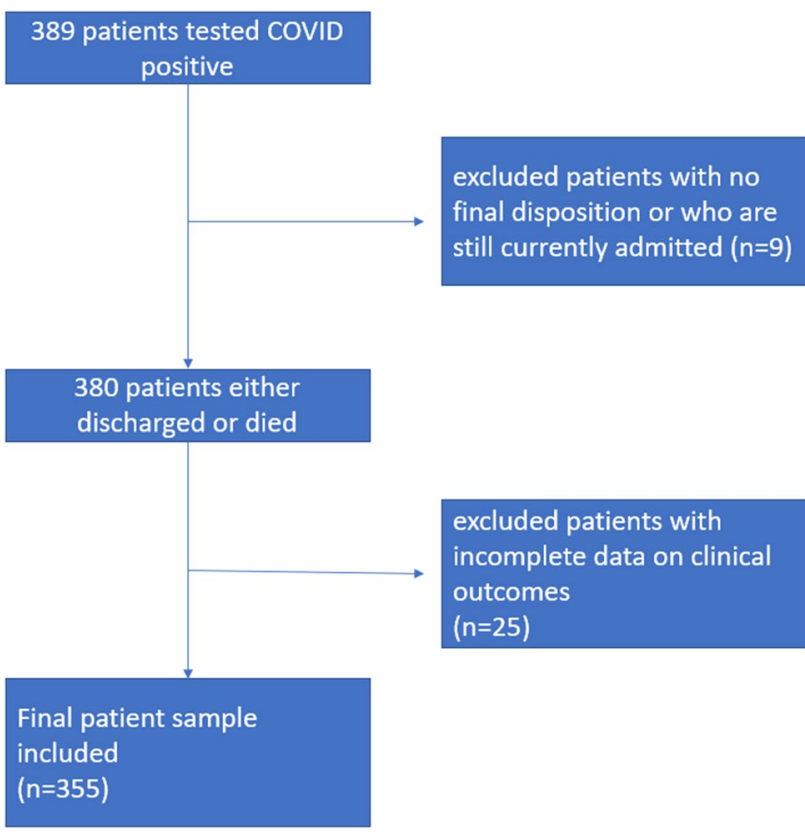

Fig. 1 Flow diagram for the study

diabetes who were given steroids ( $35 \%$ vs $24 \% p=0.026$ ), and steroid use itself was associated with significantly higher inpatient death $(47 \%$ vs $17 \% p<0.0001)$ compared to those who were not given steroids. There was also a significantly higher rate of the need for RRT/HD ( $21 \%$ vs $11 \% p=0.013)$ and the need for vasopressors ( $28 \%$ vs $18 \% p=0.023)$ among patients with diabetes compared to those without. There were higher rates of inpatient mortality among patients with diabetes, but this only showed a trend toward significance (27\% vs $19 \% p=0.053)$. After multivariate logistic regression adjusting for various demographic and comorbidity variables, only age was an independent predictor of inpatient death among patients with diabetes OR $1.03995 \%$ CI (1.003 to 1.077) $p=0.035$ (Table 2). A subgroup analysis looking at patients 60 years of age or older showed significantly higher risk of death ( $27 \%$ vs $12 \% p=0.001)$. Another subgroup analysis looking at African-American patients with diabetes shows no significant differences in terms of outcomes on inpatient mortality $p=0.856$, need for RRT/HD $p=0.429$, need for intubation $p=1.000$ and need for vasopressors $p=0.471$, compared to non-African-Americans. On univariate analysis, patients with diabetes had significantly more composite outcome of inpatient death, need for RRT/HD, vasopressors and intubation compared to those without diabetes $(44 \%$ vs $33 \% p=0.037)$. However, on multivariate regression (see Table 3 ), after adjusting for the various demographic and clinical variables, it was no longer statistically significant OR $1.495 \%$ CI (0.847 to 2.315 ) $p=0.189$.

\section{Discussion}

Looking at the prior research on the effect of type 2 diabetes on outcomes in other viral syndromes such as Middle Eastern Respiratory Syndrome (MERS) and Severe Acute Respiratory Syndrome (SARS), those with diabetes were shown to have higher mortality $[5,6]$.

On review of the current literature of the association between diabetes and COVID-19 outcomes, it has been shown that those with diabetes had significantly higher mortality as well as evidence of multi-organ injury when compared to those without diabetes. This was demonstrated by a large retrospective study done in China. The same study also demonstrated that those with well-controlled blood glucose tended to have lower mortality as well [10]. This highlights the importance of our study further characterizing the effect of diabetes on outcomes in our patient population to thus ascertain further areas of research such as association between degree of control of diabetes and subsequent outcomes.

Patients with diabetes have high rates of other metabolic risk factors including hypertension, higher BMI, higher frequency of chronic kidney disease (CKD), coronary artery disease (CAD) and heart failure, which are expected. They also tend to have higher inflammatory markers compared to those without diabetes. Obesity and related diseases such as hypertension, dyslipidemias and metabolic syndrome have been shown to have elevated inflammatory biomarkers such as interleukin-6 (IL-6) and C-reactive protein (CRP). This correlation has been linked to the development of cardiovascular disease and type 2 diabetes [11]. There is a lot of literature that has shown the chronic inflammatory state in patients with diabetes [12]. This link becomes increasingly important as in COVID-19, increased levels of certain inflammatory biomarkers have been linked to disease severity [7, 13, 14]. Pro-inflammatory cytokines and increased production of glycosylation end products can all be induced by hyperglycemia and insulin-resistant states. Patients with diabetes have a higher propensity of developing infections, and this chronic inflammatory process may be the underlying mechanism $[15,16]$.

The patients with diabetes had higher rates of need for RRT/HD as well as the need for vasopressors. There was also a trend to higher mortality. It is likely that patients with diabetes get more severe disease as evident by the above. This effect did not vary in terms of ethnicity or race, perhaps because we also had a lot of other minorities including Hispanics, Asians, etc., who are also at high risk. In fact, Caucasians were the minority in our study [17]. However, on multivariate regression and thus adjustment for clinical variables and demographics, these 
Table 1 Demographic and clinical profile of patients

\begin{tabular}{|c|c|c|c|}
\hline & Diabetes $(n=166)$ & No Diabetes $(n=189)$ & $p$ value \\
\hline Age $($ mean \pm SD) & $66.42 \pm 12.67$ & $66.03 \pm 15.46$ & 0.797 \\
\hline Female gender $n(\%)$ & $80(48)$ & $101(53)$ & 0.340 \\
\hline Ethnicity $n(\%)$ & & & 0.515 \\
\hline African-American & $118(71)$ & $134(71)$ & \\
\hline Caucasian & $10(6)$ & $17(9)$ & \\
\hline Hispanic & $21(13)$ & $17(9)$ & \\
\hline Other & $17(10)$ & $21(11)$ & \\
\hline \multicolumn{4}{|l|}{ Comorbidities } \\
\hline $\mathrm{BMI}($ mean $\pm \mathrm{SD})$ & $31.09 \pm 8.53$ & $28.53 \pm 9.43$ & 0.009 \\
\hline COPD & $22(13)$ & $23(12)$ & 0.873 \\
\hline Asthma & $11(7)$ & $16(9)$ & 0.553 \\
\hline Heart failure & $40(24)$ & $20(11)$ & 0.001 \\
\hline Atrial fibrillation & $15(9)$ & $24(13)$ & 0.310 \\
\hline Liver cirrhosis & $5(3)$ & $5(3)$ & 1.000 \\
\hline Chronic kidney disease & $42(25)$ & $23(12)$ & 0.002 \\
\hline End-stage renal disease on dialysis & $27(16)$ & $14(7)$ & 0.012 \\
\hline Coronary artery disease & $45(27)$ & $32(17)$ & 0.028 \\
\hline Hypertension & $151(91)$ & $121(64)$ & $<0.0001$ \\
\hline HIV & $3(2)$ & $4(2)$ & 1.000 \\
\hline \multicolumn{4}{|c|}{ Clinical and laboratory parameters (mean $\pm \mathrm{SD})$} \\
\hline $\mathrm{FiO} 2 \%$ requirement on admission & $28(21-44)$ & $27(21-40)$ & 0.324 \\
\hline Serum ferritin on admission $(\mathrm{ng} / \mathrm{mL})$ & $917(414-1922)$ & $802(262-1721)$ & 0.187 \\
\hline Peak ferritin $(\mathrm{ng} / \mathrm{mL})$ & $1375(594-3605)$ & $1128(354-2940)$ & 0.074 \\
\hline D-dimer on admission (ng/mL) & $2035(1062-3490)$ & $1605(820-3095)$ & 0.187 \\
\hline Peak D-dimer (ng/mL) & $3710(1633-8375)$ & $2940(1315-7923)$ & 0.302 \\
\hline $\mathrm{CRP}$ on admission $(\mathrm{mg} / \mathrm{L})$ & $143(65-230)$ & $125(50-192)$ & 0.091 \\
\hline Peak CRP (mg/L) & $184(111-258)$ & $142(65-229)$ & 0.012 \\
\hline Procalcitonin (ng/mL) & $0.28(0.10-1.13)$ & $0.18(0.08-0.68)$ & 0.080 \\
\hline Peak procalcitonin (ng/mL) & $0.46(0.11-2.79)$ & $0.28(0.10-1.19)$ & 0.065 \\
\hline LDH on admission (IU/L) & $422(310-573)$ & $397(257-537)$ & 0.146 \\
\hline Peak LDH (IU/L) & $560(384-758)$ & $499(324-655)$ & 0.017 \\
\hline \multicolumn{4}{|l|}{ COVID-19 treatment } \\
\hline Hydroxychloroquine & $100(60)$ & $116(61)$ & 0.828 \\
\hline Steroids & $58(35)$ & $45(24)$ & 0.026 \\
\hline Tocilizumab & $21(13)$ & $22(12)$ & 0.871 \\
\hline \multicolumn{4}{|l|}{ Clinical outcomes } \\
\hline Median days of hospitalization & $7(4-14)$ & $7(4-12)$ & 0.831 \\
\hline Inpatient death & $45(27)$ & $35(19)$ & 0.053 \\
\hline Need for RRT/HD & $35(21)$ & $21(11)$ & 0.013 \\
\hline Need for vasopressors & $47(28)$ & $34(18)$ & 0.023 \\
\hline Need for intubation & $48(29)$ & $41(22)$ & 0.141 \\
\hline
\end{tabular}

findings of in patient death, need for RRT/HD, vasopressor and intubation were no longer statistically significant. We suspect this is due to the interplay of other comorbidities, dilution by age as well as the relatively small sample size.

Patients who had diabetes also got more steroids which were most likely a form of selection bias where sicker people tend to get more aggressive treatment. However, this has some treatment implications such as hyperglycemia and poorer glucose control. Although temporal associations cannot truly be established in a retrospective study design, hyperglycemia itself has also been associated with poor hospital outcomes in previous studies [18, 19]. In addition, it is important to note that hyperglycemia can occur without a previous diagnosis of diabetes. The attachment of the coronavirus 2 (SARS-CoV-2) to the angiotensin converting enzyme 2 (ACE2) receptors that are present in the islet 
Table 2 Multivariate regression looking at factors associated with inpatient death among patients with diabetes and COVID-19

\begin{tabular}{lll}
\hline Characteristics & Odds ratio (95\% CI) & $p$ value \\
\hline Age & $1.039(1.003-1.077)$ & 0.035 \\
BMI & $1.001(0.950-1.055)$ & 0.962 \\
Male & Referrant & \\
Female & $0.730(0.334-1.597)$ & 0.431 \\
African-American & Referrant & \\
Caucasian & $2.726(0.594-12.504)$ & 0.197 \\
Hispanic & $0.843(0.236-3.015)$ & 0.793 \\
Others & $0.755(0.207-2.759)$ & 0.671 \\
COPD & $1.150(0.380-3.478)$ & 0.805 \\
Asthma & $0.714(0.076-6.670)$ & 0.768 \\
HF & $1.412(0.495-4.027)$ & 0.519 \\
CAD & $1.579(0.562-4.436)$ & 0.386 \\
HTN & $0.520(0.134-2.022)$ & 0.345 \\
Atrial fibrillation & $0.488(0.118-2.023)$ & 0.323 \\
CKD & $1.439(0.608-3.404)$ & 0.408 \\
\hline
\end{tabular}

Table 3 Multivariate regression looking at factors associated with composite outcome of inpatient death, need for RRT/HD, intubation and vasopressors in patients with COVID-19

\begin{tabular}{lll}
\hline Characteristics & Odds ratio $(95 \%$ CI $)$ & $p$ value \\
\hline Age & $1.017(0.997-1.038)$ & 0.095 \\
BMI & $0.992(0.963-1.021)$ & 0.992 \\
Male & Referrant & \\
Female & $1.226(0.764-1.968)$ & 0.398 \\
African-American & Referrant & \\
Caucasian & $1.337(0.524-3.412)$ & 0.543 \\
Hispanic & $0.672(0.283-1.599)$ & 0.369 \\
Others & $1.007(0.457-2.216)$ & 0.987 \\
COPD & $1.638(0.812-3.304)$ & 0.168 \\
Asthma & $0.879(0.337-2.291)$ & 0.792 \\
HF & $1.941(1.004-3.753)$ & 0.049 \\
CAD & $1.579(0.869-2.870)$ & 0.134 \\
HTN & $1.705(0.880-3.303)$ & 0.114 \\
Atrial fibrillation & $1.398(0.661-2.955)$ & 0.380 \\
CKD & $0.754(0.402-1.417)$ & 0.381 \\
Diabetes & $1.400(0.847-2.315)$ & 0.189 \\
\hline
\end{tabular}

cells of the pancreas can result in a transient hyperglycemic state [20]. Ultimately hyperglycemia, regardless of previous diabetes diagnosis, has been shown to cause more severe disease and worse outcomes [19]. This can present as a huge challenge in the efforts to achieve optimal glucose control in patients with concomitant severe COVID-19 and diabetes plus steroid use.

Interestingly, after taking into account all factors including comorbidities and demographics, among diabetic patients with COVID-19, ultimately only age was shown to be an independent predictor of mortality after multivariate regression, but not any of the cardiovascular factors.

\section{Limitations}

This study was limited by the nature of its retrospective single-center design. Medications that may influence outcomes such as those used for diabetes treatment were not taken into account. Since our institution caters to an underserved population, compliance to standard medical therapy and appropriate follow-up may be an issue and was not addressed in this study. Many of our patients did not have a documented A1c, and thus, there was an inability to do analyses by A1c and thus comparisons based on diabetic control. In addition, we did not have the detailed amount of steroid doses given to our patients which might have affected the degree of diabetes control. This does, however, highlight the necessity for further study comparing outcomes in COVID-19 based on control of diabetes. A majority of our study population were at high risk including predominantly African-Americans with multiple comorbidities, but also other minority ethnic groups like Hispanic and Asian patients. Our study gives us a glimpse into the outcomes of these high-risk population groups.

\section{Conclusion}

Our study demonstrates that patients with COVID-19 and diabetes mellitus tend to have more severe disease and poorer clinical outcomes. Only age was found to be an independent predictor of mortality. We also found that AfricanAmerican patients with diabetes did not have significant difference in outcomes and disease severity when compared to non-African-American patients.

Acknowledgements There was no monetary or material support for this research investigation.

\section{Compliance with ethical standards}

Conflict of interest None of the authors have any conflicts of interest to disclose.

Ethics Approval This study was reviewed and approved by the institutional review board of our institution. Albert Einstein Health Network (AEHN) Institutional Review Board. IRB-2020-436 Title: Demographic and Clinical Characteristics of Patients tested for COVID-19 at Einstein Medical Center Philadelphia. Date of approval 5/6/2020.

Informed Consent No informed consent was obtained as this study analyzed deidentified participant data for which formal consent is not required. 


\section{References}

1. Kumar A, Arora A, Sharma P et al (2020) Is diabetes mellitus associated with mortality and severity of COVID-19? A metaanalysis. Diabetes Metab Syndr 14(4):535-545. https://doi. org/10.1016/j.dsx.2020.04.044

2. Ahn DG, Shin HJ, Kim MH et al (2020) Current status of epidemiology, diagnosis, therapeutics, and vaccines for novel coronavirus disease 2019 (COVID-19). J Microbiol Biotechnol 30(3):313324. https://doi.org/10.4014/jmb.2003.03011

3. Centers for Disease Control and Prevention (2020) Daily confirmed, and probable COVID-19 infections and deaths in the U.S. Retrieved from https://www.cdc.gov/coronavirus/2019-ncov/cases -updates/cases-in-us.html

4. World Health Organisation (2020) WHO Coronavirus disease (COVID-19) Dashboard. Retrieved from https://covid19. who.int

5. Morra ME, Van Thanh L, Kamel MG et al (2018) Clinical outcomes of current medical approaches for Middle East respiratory syndrome: a systematic review and meta-analysis. Rev Med Virol 28(3):e1977. https://doi.org/10.1002/rmv.1977

6. Chan JW, Ng CK, Chan YH et al (2003) Short term outcome and risk factors for adverse clinical outcomes in adults with severe acute respiratory syndrome (SARS). Thorax 58(8):686-689. https ://doi.org/10.1136/thorax.58.8.686

7. Zhou F, Yu T, Du R, Fan G, Liu Y, Liu Z (2020) Clinical course and risk factors for mortality of adult inpatients with COVID-19 in Wuhan, China: a retrospective cohort study. Lancet 395:10541062. https://doi.org/10.1016/S0140-6736(20)30566-3

8. Guan WJ, Liang WH, Zhao Y et al (2020) Comorbidity and its impact on 1590 patients with COVID-19 in China: a nationwide analysis. Eur Respir J. 55(5):2000547. https://doi. org/10.1183/13993003.00547-2020

9. Williams R, Karuranga S, Malanda B, Saeedi P, Basit A, Besançon $S$ (2020) Global and regional estimates and projections of diabetes-related health expenditure: results from the International Diabetes Federation Diabetes Atlas. Diabetes Res Clin Pract. https ://doi.org/10.1016/j.diabres.2020.108072

10. Zhu L, She ZG, Cheng X et al (2020) Association of blood glucose control and outcomes in patients with COVID-19 and preexisting type 2 diabetes. Cell Metab 31(6):1068-1077.e3. https:// doi.org/10.1016/j.cmet.2020.04.021

11. Pradhan AD, Manson JE, Rifai $\mathrm{N}$ et al (2001) C-reactive protein, interleukin 6 , and risk of developing type 2 diabetes mellitus. JAMA 286:327-334. https://doi.org/10.1001/jama.286.3.327
12. Tsalamandris S, Antonopoulos AS, Oikonomou E, Papamikroulis G-A, Vogiatzi G, Papaioannou S (2019) The role of inflammation in diabetes: current concepts and future perspectives. Eur Cardiol 14:50. https://doi.org/10.15420/ecr.2018.33.1

13. Guo W, Li M, Dong Y, Zhou H, Zhang Z, Tian C (2020) Diabetes is a risk factor for the progression and prognosis of COVID-19. Diabetes Metab Res Rev. https://doi.org/10.1002/dmrr.3319

14. Zheng F, Tang W, Li H, Huang Y-X, Xie Y-L, Zhou Z-G (2020) Clinical characteristics of 161 cases of corona virus disease 2019 (COVID-19) in Changsha. Eur Rev Med Pharmacol Sci 24:34043410. https://doi.org/10.26355/eurrev_202003_20711

15. Sylvia K (2013) Diabetes and infection: is there a link? - a minireview. Gerontology 59(2):99-104. https://doi.org/10.1159/00034 5107

16. Petrie JR, Guzik TJ, Touyz RM (2018) Diabetes, hypertension, and cardiovascular disease: clinical insights and vascular mechanisms. Can J Cardiol 34(5):575-584. https://doi.org/10.1016/j. cjca.2017.12.005

17. Devakumar D, Shannon G, Bhopal SS, Abubakar I (2020) Racism and discrimination in COVID-19 responses. Lancet 395(10231):1194. https://doi.org/10.1016/S0140-6736(20)30792 $-3$

18. Bode B, Garrett V, Messler J, McFarland R, Crowe J, Booth R, et al (2020) Glycemic characteristics and clinical outcomes of COVID-19 patients hospitalized in the United States. J Diabetes Sci Technol (in press). https://www.bloomberg.com/press -releases/2020-04-17/covid-19-study-shows-more-than-4-times -in-hospital-mortality-rate-and-increased-length-of-stay-for-patie nts-with-diabetes-and

19. Iacobellis G, Penaherrera CA, Bermudez LE, Bernal ME (2020) Admission hyperglycemia and radiological findings of SARSCoV2 in patients with and without diabetes. Diabetes Res Clin Pract 164:108185. https://doi.org/10.1016/j.diabres.2020.108185

20. Yang JK, Lin SS, Ji XJ, Guo LM (2010) Binding of SARS coronavirus to its receptor damages islets and causes acute diabetes. Acta Diabetol 47:193-199. https://doi.org/10.1007/s00592-009-0109-4

Publisher's Note Springer Nature remains neutral with regard to jurisdictional claims in published maps and institutional affiliations. 